\title{
Una aproximación a los conceptos de emprendedor y emprendimiento social
}

\author{
María Luisa Saavedra García* \\ María Elena Camarena Adame ${ }^{\star \star}$ \\ Mario Enrique Vargas Saenz ${ }^{* *}$
}

Fecha de recibido: 6 de junio de 2019 Fecha de aprobado: 29 de enero de 2020

\begin{abstract}
Para citar este artículo: Saavedra García, M. L., Camarena Adame, M. E., \& Vargas Saenz, M. E. (2020) Una aproximación a los conceptos de emprendedor y emprendimiento social. Revista Universidad \& Empresa, 22(39), 1-27. https://doi. org/10.12804/revistas.urosario.edu.co/empresa/a.7976
\end{abstract}

\section{Resumen}

El emprendedor y el emprendimiento social como temas de investigación en Latinoamérica se encuentran aún en una etapa inicial, y no hay un consenso acerca de su conceptualización, por esta razón este trabajo tiene como objetivo analizar los conceptos que se han vertido acerca del emprendedor y el emprendimiento social, con el fin de servir de marco de referencia para posteriores estudios y aportar a la mejor comprensión de este fenómeno. Se utilizó el método de investigación documental, realizando una revisión de la literatura de trabajos de investigación publicados en revistas científicas arbitradas. Los principales hallazgos permiten identificar las variables principales contenidas en ambos conceptos, las cuales resultan de utilidad para investigaciones futuras.

Palabras Clave: emprendedor social; emprendimiento social; empresa social.

* Profesora titular de tiempo completo en la UNAM, Facultad de Contaduría y Administración, División de Investigación. Correo electrónico: Isaavedra@fca.unam.mx

** Profesora titular de tiempo completo en la UNAM, Facultad de Contaduría y Administración, División de Investigación. Correo electrónico: mcamarena_adame@hotmail.com

*** Licenciado en Folosofía y Letras y Magister en Administración por la Uiversidad EAFIT, Doctor en Filosofia por la Universitá Pontificia Salesiana.Director de EAFIT Social, Universidad EAFIT, Medellín Colombia. Correo electrónico: mvargas@eafti.edu.co 


\title{
An Approach to the Concepts of Entrepreneurship and Social Entrepreneurship
}

\begin{abstract}
The entrepreneur and social entrepreneurship as research topics in Latin America are still in an initial stage, and there is no consensus about its conceptualization. For this reason, this work aims to analyze the concepts of entrepreneur and social entrepreneurship, in order to serve as a reference for further studies and provide greater understanding of this phenomenon. The method used was a documentary research, carrying out a literature review of research papers published in peer-reviewed scientific journals. The main findings allow identifying the main variables contained in both concepts, which are useful for future research.
\end{abstract}

Keywords: Social entrepreneur; social entrepreneurship; social enterprise.

\section{Uma aproximação aos conceitos de empreendedor e empreendimento social}

\section{Resumo}

O empreendedor e o empreendimento social como temas de pesquisa na América Latina, encontram-se ainda em uma etapa inicial, não existe um consenso de sua conceitualização, é por esta razão que este trabalho tem como objetivo analisar os conceitos que se têm vertido acerca do empreendedor e o empreendimento social, com o fim de servir de marco de referência para posteriores estudos e aportar maior compreensão a este fenômeno. Utilizou-se o método de pesquisa documental, realizando uma revisão da literatura de trabalhos de pesquisa pulicados em revistas científicas arbitradas. Os principais resultados permitem identificar as variáveis principais contidas em ambos os conceitos, os quais resultam de utilidade para pesquisas futuras.

Palavras-chave: empreendedor social; empreendimento social; empresa social.

\section{Introducción}

El emprendimiento social ha surgido como un nuevo fenómeno que está ayudando a mejorar las condiciones de vida de muchas personas alrededor del mundo (Felix, Husted \& Aigner, 2017). De acuerdo con Rey, Ribeiro y Sánchez (2016) el término social se refiere al valor que una empresa agrega a la sociedad en tres áreas: (1) beneficio económico para la sociedad; (2) beneficio ecológico, cuidado del medio ambiente, y (3) beneficio social, calidad de vida. Por su parte, Seham, Ahmed y Ayman (2017) han señalado que entre las personas que inician emprendimientos sociales se encuentran empresarios que toman riesgos y son compasivos, con mentalidades emprendedoras que buscan abordar problemas sociales de maneras innovadoras. También tienen la perseverancia para enfrentar los ineficientes marcos institucionales prevalecientes en las economías en desarrollo. Los 
emprendedores sociales están motivados por problemas y desafíos sociales, inspiración y experiencias personales previas, así como por sus redes sociales.

El interés actual por el emprendimiento social es consistente con el aumento de la demanda impuesta a las organizaciones empresariales de desencadenar un cambio social positivo mediante la participación social e iniciativas medioambientales (Aguilera et al. 2007; Bronn \& Vidaver-Cohen 2009).

Un aspecto importante a destacar es que al involucrar el sector social en esos emprendimientos se fortalecen los niveles de competitividad empresarial, que contribuyen al desarrollo de la región o del país. Esto demuestra que las empresas creadas con enfoque social generan no solo empleos y una concepción diferente de empresa, sino que también brindan bienestar a la comunidad involucrada, reduciendo los niveles de exclusión social (Salinas \& Osorio, 2012).

Autores como Thompson, Alvy y Lees (2000); Mair y Schoen (2007); Zahra et al. (2009); Austin, Stevenson y Wei-Skillern (2006); Dacin, Dacin y Tracey (2011); Dees (2012), y Pache y Santos (2013) han reconocido el emprendimiento social como un campo importante de investigación. Sin embargo, a pesar de la existencia de una amplia atención global a este tipo de emprendimiento, la teoría sobre este todavía está en la etapa de conceptualización, ya que cada país tiene diferentes aspectos específicos de la cobertura del emprendimiento social y actitudes hacia las iniciativas de emprendimiento. Es por esta razón que este trabajo tiene como objetivo analizar los conceptos que se han vertido acerca del emprendedor y el emprendimiento social, con el fin de servir de marco de referencia para posteriores estudios y aportar a la mejor comprensión de este fenómeno. La investigación es de carácter documental, se revisó la literatura en torno a los conceptos de empresa social, emprendimiento social y emprendedor social; los principales hallazgos se muestran en las conclusiones. 


\section{Empresa social}

Una de las primeras nociones de empresa social fue definida por Talbot, Tregilgas y Harrison (2002):

medio por el cual las personas se reúnen y utilizan las empresas basadas en el mercado para lograr un acuerdo de fines sociales. Se caracteriza por la creatividad, el espíritu empresarial y un enfoque en la comunidad más que de beneficio individual. Es un esfuerzo creativo que resulta en servicios sociales, financieros, de servicio, beneficios educativos, de empleo u otros beneficios comunitarios (p. 2).

Silva, Botero y Soto (2014) señalan que es aquella que tiene un fin social desde que nace, se transforma, produce y se relaciona con los trabajadores teniendo presente este fin; sea cual sea la figura jurídica que asuma, sus características se encuentran ligadas a las dimensiones económica y social. En lo económico, estas empresas:

- Deben producir bienes y servicios de modo continuo y en constante relación con el mercado.

- Son autónomas y definen de modo independiente la dirección que tomarán.

- Cuentan con personal remunerado, sin dejar de lado el apoyo de personal voluntario.

- Se arriesgan al diversificar productos y servicios, buscando nuevos canales de distribución con el objetivo de ampliar su cartera de clientes.

- Buscan la innovación ofreciendo un servicio nuevo o probando nuevas modalidades.

En lo social:

- Nacen bajo la iniciativa de un grupo de personas que persiguen un fin común.

- Contribuyen de manera significativa al desarrollo social de un territorio.

- Toman decisiones sin tener en cuenta la cantidad aportada por cada socio (no tiene más poder, el quien aporta más).

- Tienen un modelo de gestión democrático y participativo (una cabeza, un voto). 
- Explicitan su finalidad social.

Por su parte, Yunus (2012) ha señalado que una empresa social es un negocio autosostenible que no genera pérdidas, pero tampoco reparte dividendos; asume un objetivo social según las normas que rigen en el mercado; es decir, genera suficientes ingresos que garantizan su funcionamiento y reinvierten las utilidades, en su objetivo social buscan un impacto positivo en la sociedad al fortalecer la inclusión social de personas en situación de vulnerabilidad.

Es necesario aclarar que una empresa social no es una organización sin fines de lucro, pues estas no recuperan sus costos de operación y se fondean a través de subsidios del gobierno y donaciones de terceros. En cambio, la empresa social debe no solo proveer bienes y servicios que cumplen un objetivo social, sino que también debe ser capaz de recuperar sus costos.

Según Yunus (2012), las características principales de una empresa social son

- Tener como objetivo abatir la pobreza o atender uno o más problemas sociales, no la maximización de utilidades.

- Contar con sostenibilidad financiera y económica.

- Recuperar la inversión inicial y no gozar de dividendos.

- Reinvertir las utilidades para mejorar y ampliar la empresa.

- Ser responsable con el medioambiente

- Fijar los salarios de los trabajadores de acuerdo con los fijados por el mercado y condiciones laborales que superen el estándar.

- Ser creadas con alegría.

Una definición más reciente, adoptada por sIEe (2014), identifica a las empresas sociales así:

- Son impulsadas por una misión económica, social, cultural o ambiental para el beneficio público.

- Ubican a las personas en el centro de sus negocios.

- Comercian de manera rentable para cumplir con su misión.

- Reinvierten las ganancias o excedentes en el cumplimiento de su misión. 
- Involucran a un amplio grupo de partes interesadas en el gobierno y la toma de decisiones.

- Operan con transparencia.

La Unión Europea (2014), por su parte, ha señalado que la empresa social tiene como objetivo principal impactar en la sociedad, más que generar utilidades para los socios; opera como empresa en el mercado proporcionando bienes y servicios de manera innovadora, utilizando los excedentes para fines sociales, realizando una gestión responsable y transparente.

Un aspecto a destacar, referido por Melián y Campos (2010) y Kostetska y Berezyak, (2014), es que las empresas de la economía social surgen principalmente de la innovación social, la cual no es otra cosa que nuevas formas de organización y relaciones laborales basadas en el establecimiento de fuertes relaciones sociales, tanto con los clientes como con los trabajadores.

Con base en lo anterior, se puede decir que la empresa social tiene como fin dar solución a un problema social, basándose en la innovación social y buscando la sostenibilidad financiera.

\subsection{Emprendimiento social}

El emprendimiento social es un fenómeno ampliamente reconocido por su capacidad de generar valor social (Danko, Brunner \& Kraus, 2011). Bill Drayton fundador de Ashoka, fue el que popularizó el término emprendimiento social a finales de los noventas y con ello ha impulsado a varios emprededores sociales alrededor del mundo; antes de él Michael Young promovió la idea del emprendedor social entre 1950 y 1990. Sin embargo, el concepto era conocido implícitamente desde antes, desde el siglo xix se han fundado empresas con fines sociales (Seham et al., 2017). Así pues, los emprendedores sociales han existido de tiempo atrás por ejemplo, Florence Nightingale fundó la primera escuela de enfermería y desarrolló prácticas modernas en dicho campo; Robert Owen, conocido por su modelo cooperativista de negocios, es uno de los emprendedores sociales más famosos de la historia. 
El emprendimiento se define como un proceso dinámico de visión, cambio y creación que requiere la aplicación de energía y pasión para la implementación de nuevas ideas y soluciones creativas (Kuratko, 2005). Este fenómeno se entiende como el conjunto de actitudes y conductas que dan un perfil asociado a aspectos como el control a situaciones de riesgo, la creatividad para plantear propuestas, la capacidad de innovación, la autoconfianza y la toma de decisiones, a estas características se les denomina: acción emprendedora. Esta es una acción innovadora que, a través de un sistema organizado de relaciones interpersonales y la combinación de recursos, se orienta al logro de un determinado fin, se asocia con la creación de algo nuevo y la generación de un nuevo valor, producto, bien o servicio que anterior a la acción no existía (Selamé, 1999).

Gartner (1985) considera cuatro dimensiones generalmente aceptadas de creación de empresas emprendedoras: individuo, organización, proceso y medio ambiente. En cuanto a las características individuales considera la necesidad de logro, el locus de control, la propensión a tomar riesgos, la satisfacción por el trabajo, la experiencia laboral previa, los padres emprendedores, la edad y la educación. En lo que se refiere a la organización, incluye el liderazgo en costos, la diferenciación, el enfoque, los nuevos productos o servicios, la competencia paralela, la entrada de franquicias, la transferencia geográfica, la escasez de suministros, el aprovechamiento de recursos no utilizados, el contrato del cliente, convertirse en una segunda fuente, las empresas conjuntas, las licencias, la renuncia al mercado, el vender fuera de la división, las compras preferidas por el gobierno y los cambios en las reglas gubernamentales. En la dimensión de medio ambiente incorpora la presencia de empresarios experimentados; la fuerza de trabajo técnicamente calificada; la accesibilidad a proveedores, clientes o nuevos mercados y transporte; la influencia del gobierno, la proximidad de universidades, de terrenos o instalaciones, servicios de soporte y recursos financieros; la actitud de la población del área; las condiciones de vida; la alta diferenciación ocupacional e industrial; los altos porcentajes de inmigrantes recientes en la población; la gran base industrial; las áreas urbanas de mayor tamaño; las barreras para entrar; la rivalidad entre los competidores existentes; la presión de productos sustitutos, y el poder de negociación de los compradores y proveedores. Por último, en lo referente al proceso se tiene en cuenta al emprendedor quien encuentra una oportunidad de negocio, acumula recursos, comercializa productos y servicios, produce el producto, construye una organización y responde al gobierno y la sociedad. A este respecto, Sekliuckiene y 
Kisielius (2015) han encontrado que las circunstancias y el contexto socioempresarial son los factores más importantes para todas las etapas del proceso de emprendimiento social.

Posteriormente, el propio Gartner (1989) propone ocho factores que componen la definición de emprendimiento: persona, innovación, organización, creación, valor, ganancias, originalidad y dueño-administrador. Además, establece que lo importante no son las características del emprendedor sino el proceso de creación de la organización o negocio el cual finaliza al tiempo que se crea dicha organización. El emprendimiento tiene temporalidad, no es un estado permanente, ya que considera que esta acción depende de las coyunturas que cada proceso de integración organizacional posee, el emprendimiento está en la acción del emprendedor.

Por su parte, Silva, Botero y Soto (2014) sugieren que el emprendimiento es aquella actitud y aptitud de la persona que le permiten comenzar nuevos retos o proyectos; es lo que le permite avanzar un paso más, ir más allá de donde ya ha llegado.

En lo que corresponde al emprendimiento social, este se refiere a las iniciativas que se realizan bajo tres ejes: 1) un objetivo social, 2) un modelo de negocio sostenible y 3) una forma innovadora de abordar un problema social (Silva, Botero \& Soto, 2014).

De acuerdo con Rodríguez (2007):

frente a] la reacción a la desigualdad y la ausencia de oportunidades reales de desarroIlo, surgen liderazgos que no esperan la atención de programas sociales asistenciales, sino que buscan dentro de las potencialidades de la comunidad una alternativa al problema, que involucre la creación de valor social. Para algunos, las grandes deudas sociales del Estado (p. 15).

Los emprendimientos sociales tienen características en común, aunque cada uno tiene las propias dependiendo del grupo social que lo realice y de las condiciones donde se ubique. A continuación, se definen cuatro aspectos diferenciadores del emprendimiento (Curto, 2012): 
- Destrucción creativa: la generación de productos o servicios innovadores destruye a los que estaban en el mercado.

- Creación de valor: traspasar recursos económicos de un área de baja productividad a una de alta y mayor rendimiento.

- Identificación de oportunidades: explotar las oportunidades que los cambios brindan.

- Ingenio: la capacidad de no solo aprovechar oportunidades, sino también de afrontar los retos que implican la falta de recursos para llevarlas a cabo.

En suma, el emprendimiento social cristalizado en las empresas sociales identifica las necesidades existentes en un territorio con el fin de transformarlas en oportunidades empresariales para mediante la producción continua de bienes y servicios contribuir con el bienestar colectivo de la comunidad (Silva, Botero \& Soto, 2014).

Por su parte, Rodríguez (2016) sostiene que el emprendimiento social es parte del emprendimiento sostenible. El aspecto social del emprendimiento sostenible tiene que ver con el comportamiento de las empresas en aspectos sociales y éticos como la gestión del recurso humano, los derechos humanos, el trabajo infantil, el género, la discriminación, la participación de los trabajadores en beneficios de la empresa y la corrupción. Lo medioambiental implica productos limpios, ecoeficiencia, desarrollo tecnológico sostenible, y ecodiseño. El tercer aspecto tiene que ver con los resultados financieros de la empresa.

Conway y Kalakay (2016) señalan que se han realizado importantes investigaciones para definir el emprendimiento social. Sin embargo, muchos esfuerzos no exploran la dimensionalidad completa del concepto, ya que sus hallazgos revelan que no hay definición. Estos autores exploraron las cuatro dimensiones del espíritu empresarial (individuo, entorno, proceso y organización) postuladas por Gartner (1985), y encontraron un avance limitado en el desarrollo de las definiciones.

A continuación, se presenta una revisión del concepto de emprendimiento social. 
Tabla 1. Conceptos de emprendimiento social

\begin{tabular}{|c|c|c|}
\hline Autor/año & Concepto & Variables \\
\hline Leadbeater (1997) & $\begin{array}{l}\text { Uso de la función empresarial con fines sociales y no para obtener } \\
\text { beneficios o alternativamente que los beneficios generados por las } \\
\text { actividades de mercado se utilizan en beneficio de un grupo desfavo- } \\
\text { recido particular. }\end{array}$ & $\begin{array}{l}\text { - } \text { Fines sociales } \\
\text { - Cubre necesida- } \\
\text { des }\end{array}$ \\
\hline Fowler (2000) & $\begin{array}{l}\text { Es la creación de estructuras, relaciones, instituciones, organizaciones } \\
\text { y prácticas socioeconómicas viables que generan y sostienen benefi- } \\
\text { cios sociales. }\end{array}$ & $\begin{array}{l}\text { - Prácticas socioeco- } \\
\text { nómicas } \\
\text { - Beneficios sociales }\end{array}$ \\
\hline Johnson (2000) & $\begin{array}{l}\text { Es un enfoque innovador para hacer frente a las necesidades sociales } \\
\text { complejas, con énfasis en la resolución de problemas sociales y la } \\
\text { innovación social. }\end{array}$ & $\begin{array}{l}\text { - Innovación } \\
\text { - Cubre necesida- } \\
\text { des }\end{array}$ \\
\hline $\begin{array}{l}\text { Canadian Centre } \\
\text { for Social Entrepre- } \\
\text { neurship (2001) }\end{array}$ & $\begin{array}{l}\text { Iniciativas innovadoras con la doble finalidad de asegurar que la } \\
\text { inversión genera tanto rentabilidad social como económica, desarro- } \\
\text { llados por sectores privados, públicos y voluntarios. }\end{array}$ & $\begin{array}{ll}\text { - Innovación } \\
\text { - Rentabilidad social } \\
\text { - Voluntarios }\end{array}$ \\
\hline $\begin{array}{l}\text { Smallbone et al. } \\
\text { (2001) }\end{array}$ & $\begin{array}{l}\text { Empresas sociales definidas como empresas competitivas que actúan } \\
\text { y comercian con un fin social. }\end{array}$ & $\begin{array}{l}\text { - Competitivas } \\
\text { - Fin social }\end{array}$ \\
\hline $\begin{array}{l}\text { Hibbert, Hogg y } \\
\text { Quinn (2002) }\end{array}$ & $\begin{array}{l}\text { El uso de la actividad empresarial con fines sociales y no para obtener } \\
\text { beneficios o una empresa que genera ganancias que benefician a un } \\
\text { grupo desfavorecido específico. }\end{array}$ & $\begin{array}{l}\text { - } \quad \text { Fin social } \\
\text { - Beneficios sociales }\end{array}$ \\
\hline $\begin{array}{l}\text { Cook, Dodds y } \\
\text { Mitchell (2003) }\end{array}$ & $\begin{array}{l}\text { Asociaciones con fines sociales entre los sectores público, social } \\
\text { y empresarial diseñadas para aprovechar el poder de mercado en } \\
\text { beneficio del interés público. }\end{array}$ & $\begin{array}{l}\text { - } \\
\text { - } \text { Bin social } \\
\text { - } \text { Aprovecha meciales } \\
\text { cado }\end{array}$ \\
\hline MacMillan (2003) & $\begin{array}{l}\text { Proceso mediante el cual la creación de una nueva empresa conduce } \\
\text { a la mejora de la riqueza social tanto para la sociedad como para el } \\
\text { beneficio empresarial. }\end{array}$ & $\begin{array}{l}\text { - } \\
\text { - } \\
\text { Beneficicios sociales } \\
\text { y empresariales }\end{array}$ \\
\hline Schwab (2014) & $\begin{array}{l}\text { El trabajo de las organizaciones comunitarias, voluntarias y públicas, } \\
\text { así como las empresas privadas que trabajan para el desarrollo social } \\
\text { y no solo para obtener beneficios. }\end{array}$ & $\begin{array}{l}\text { - Beneficio social } \\
\text { - Esfuerzo comuni- } \\
\text { tario }\end{array}$ \\
\hline $\begin{array}{l}\text { Fuqua School } \\
(2005)\end{array}$ & $\begin{array}{l}\text { El arte de llevar a cabo de forma simultánea el retorno de la inversión } \\
\text { tanto financiera como social. }\end{array}$ & $\begin{array}{l}\text { - Retorno de la } \\
\text { inversión social }\end{array}$ \\
\hline Haugh (2005) & $\begin{array}{l}\text { Actividades asociadas con la percepción de oportunidades para } \\
\text { crear valor social y la creación de organizaciones sociales con el pro- } \\
\text { pósito de llevarlo a cabo. }\end{array}$ & $\begin{array}{l}\text { - Creación de valor } \\
\text { social } \\
\text { - Oportunidades } \\
\text { para el cambio } \\
\text { social }\end{array}$ \\
\hline $\begin{array}{l}\text { Roberts y Woods } \\
\text { (2005) }\end{array}$ & $\begin{array}{l}\text { Es la construcción, evaluación y persecución de oportunidades para } \\
\text { el cambio social transformativo llevado a cabo por individuos visiona- } \\
\text { rios, apasionados y dedicados. }\end{array}$ & $\begin{array}{l}\text { - Oportunidades } \\
\text { para el cambio } \\
\text { social } \\
\text { - Individuos visio- } \\
\text { narios }\end{array}$ \\
\hline
\end{tabular}




\begin{tabular}{|c|c|c|}
\hline Autor/año & Concepto & Variables \\
\hline Seelos y Mair (2005) & $\begin{array}{l}\text { Organizaciones que han creado modelos para la resolución eficiente } \\
\text { de las necesidades humanas básicas, que los mercados y las institu- } \\
\text { ciones existentes no han podido satisfacer. El emprendimiento social } \\
\text { combina el ingenio del espíritu empresarial tradicional con la misión } \\
\text { de cambiar la sociedad. }\end{array}$ & $\begin{array}{l}\text { - Satisfacción de ne- } \\
\text { cesidades básicas } \\
\text { - Cambiar la socie- } \\
\text { dad }\end{array}$ \\
\hline De Pablo (2005) & $\begin{array}{l}\text { Es el compromiso de crear nuevos modelos de actividad para desa- } \\
\text { rrollar productos y servicios que satisfagan las necesidades básicas } \\
\text { de colectivos desatendidos por las instituciones sociales y económi- } \\
\text { cas convencionales. }\end{array}$ & $\begin{array}{l}\text { - Modelos empresa- } \\
\text { riales } \\
\text { - Satisfacción de ne- } \\
\text { cesidades básicas }\end{array}$ \\
\hline $\begin{array}{l}\text { Tan, Williams y Tan } \\
\text { (2005) }\end{array}$ & $\begin{array}{l}\text { La obtención de beneficios a través de la innovación asumiendo el } \\
\text { riesgo con la participación de un segmento de la sociedad, cuyos } \\
\text { beneficios o parte de ellos se depositan en ese mismo segmento de } \\
\text { la sociedad. }\end{array}$ & $\begin{array}{l}\text { - Innovación } \\
\text { - Riesgo } \\
\text { - Sociedad } \\
\text { - Beneficio social }\end{array}$ \\
\hline Austin et al. (2006) & $\begin{array}{l}\text { Una actividad innovadora, de creación de valor social, que ocurre al } \\
\text { interior y a través de los sectores sin ánimo de lucro, de negocios y } \\
\text { gubernamental. }\end{array}$ & $\begin{array}{l}\text { - Innovación } \\
\text { - Creación de valor } \\
\text { social }\end{array}$ \\
\hline $\begin{array}{l}\text { Korosec y Berman } \\
(2006)\end{array}$ & $\begin{array}{l}\text { La actividad de los individuos y las organizaciones privadas de tomar la } \\
\text { iniciativa para hacer frente a los desafíos sociales en sus comunidades. }\end{array}$ & $\begin{array}{l}\text { - Satisfacción de ne- } \\
\text { cesidades básicas }\end{array}$ \\
\hline Mair y Marti (2006) & $\begin{array}{l}\text { Modelos innovadores de proveer productos y servicios que se } \\
\text { adaptan a las necesidades básicas insatisfechas por las instituciones } \\
\text { políticas o económicas. }\end{array}$ & $\begin{array}{l}\text { - Innovación } \\
\text { - Satisfacción de ne- } \\
\text { cesidades básicas }\end{array}$ \\
\hline $\begin{array}{l}\text { Mair y Noboa } \\
(2006)\end{array}$ & $\begin{array}{l}\text { Uso innovador de combinaciones de recursos para aprovechar las } \\
\text { oportunidades con el objetivo de crear organizaciones o prácticas } \\
\text { que produzcan y mantengan beneficios sociales. }\end{array}$ & $\begin{array}{l}\text { - Innovación } \\
\text { - Oportunidades } \\
\text { - } \text { Beneficios sociales }\end{array}$ \\
\hline Nichols (2006) & $\begin{array}{l}\text { Implica innovaciones destinadas a mejorar el bienestar social de } \\
\text { forma explícita. Este se encuentra dentro de organizaciones empre- } \\
\text { sariales que inician, dirigen o contribuyen al cambio en la sociedad. }\end{array}$ & $\begin{array}{l}\text { - Innovación } \\
\text { - Bienestar social } \\
\text { - Cambio social }\end{array}$ \\
\hline $\begin{array}{l}\text { Peredo y McLean } \\
\text { (2006) }\end{array}$ & $\begin{array}{l}\text { Ejercido por alguna persona o grupo con el objetivo de crear valor } \\
\text { social, que demuestra una capacidad para reconocer y aprovechar las } \\
\text { oportunidades, emplear la innovación, aceptar un riesgo superior a la } \\
\text { media y con soluciones inusualmente ingeniosas en la búsqueda de } \\
\text { su emprendimiento social. }\end{array}$ & $\begin{array}{l}\text { - Valor social } \\
\text { - Oportunidades } \\
\text { - Innovación } \\
\text { - Riesgo }\end{array}$ \\
\hline $\begin{array}{l}\text { Weerawardena y } \\
\text { Sullivan (2006) }\end{array}$ & $\begin{array}{l}\text { Fenómeno conductual expresado en el contexto de una organización } \\
\text { no lucrativa que busca entregar un valor social a través de la explo- } \\
\text { tación de las oportunidades percibidas. El emprendimiento social se } \\
\text { esfuerza por lograr la creación de valor social y esto requiere la visua- } \\
\text { lización de la capacidad de innovación, la proactividad y el comporta- } \\
\text { miento de la gestión de riesgos. Este comportamiento se ve limitado } \\
\text { por el deseo de lograr la misión social y mantener la sostenibilidad } \\
\text { de la organización existente. }\end{array}$ & $\begin{array}{l}\text { - } \text { Valor y misión } \\
\text { - Oporial } \\
\text { - Creación de valor } \\
\text { - } \text { Innocial } \\
\text { - Proactividad } \\
\text { - Riesgos } \\
\text { - Sostenibilidad }\end{array}$ \\
\hline
\end{tabular}




\begin{tabular}{|c|c|c|}
\hline Autor/año & Concepto & Variables \\
\hline Chell (2007) & $\begin{array}{l}\text { Es crear y aprovechar las oportunidades sin descanso, sin tener en } \\
\text { cuenta los recursos enajenables controlados actualmente, con vistas } \\
\text { a la creación de riqueza, que puede ser reinvertida en el negocio } \\
\text { para asegurar su sostenibilidad, y al valor social. }\end{array}$ & $\begin{array}{l}\text { - Oportunidades } \\
\text { - Sostenibilidad } \\
\text { - Valor social }\end{array}$ \\
\hline $\begin{array}{l}\text { Martin y Osberg } \\
\text { (2007) }\end{array}$ & $\begin{array}{l}\text { El emprendimiento social está conformado por tres componentes: } \\
\text { (1) identificación de equilibrio estable pero injusto, (2) identificación de } \\
\text { oportunidad que desarrolle una proposición de valor social y (3) crea- } \\
\text { ción de un nuevo equilibrio estable que mejora al grupo objetivo. }\end{array}$ & $\begin{array}{l}\text { - } \\
\text { - Opuilibrio } \\
\text { - Valor y beneficio } \\
\text { social }\end{array}$ \\
\hline $\begin{array}{l}\text { Guzmán y Trujillo } \\
\text { (2008) }\end{array}$ & $\begin{array}{l}\text { El emprendimiento social es un tipo específico de emprendimiento } \\
\text { que busca soluciones para problemas sociales a través de la cons- } \\
\text { trucción, evaluación y búsqueda de oportunidades que permitan la } \\
\text { generación de valor social sostenible, alcanzando equilibrios nuevos y } \\
\text { estables en relación con las condiciones sociales, a través de la acción } \\
\text { directa llevada a cabo por organizaciones sin ánimo de lucro, empresas } \\
\text { u organismos gubernamentales. Busca la sostenibilidad financiera. }\end{array}$ & $\begin{array}{l}\text { - } \text { Beneficio y valor } \\
\text { - Oportunidad } \\
\text { - Equilibrio } \\
\text { - Sostenibilidad } \\
\text { financiera }\end{array}$ \\
\hline Thompson (2008) & $\begin{array}{l}\text { Es una actividad emprendedora con una orientación e intención } \\
\text { social. }\end{array}$ & - Orientación social \\
\hline Zahra et al. (2009) & $\begin{array}{l}\text { Abarca las actividades y procesos realizados para descubrir, definir y } \\
\text { aprovechar las oportunidades para mejorar la riqueza social median- } \\
\text { te la creación de nuevas empresas o la gestión de las organizaciones } \\
\text { existentes de una manera innovadora. }\end{array}$ & $\begin{array}{l}\text { - Oportunidades } \\
\text { - Riqueza social } \\
\text { - Innovación }\end{array}$ \\
\hline $\begin{array}{l}\text { Lepoutre et al } \\
\text { (2011) }\end{array}$ & $\begin{array}{l}\text { Es la actividad empresarial que tiene por objetivo hacer frente a los } \\
\text { problemas de la sociedad. }\end{array}$ & - Beneficio social \\
\hline $\begin{array}{l}\text { Tukamushaba, } \\
\text { Orobia, } \\
\text { y George (2011) }\end{array}$ & $\begin{array}{l}\text { Es la aplicación de enfoques innovadores } \\
\text { para la solución de problemas sociales. }\end{array}$ & $\begin{array}{l}\text { - Innovación } \\
\text { - Beneficio social }\end{array}$ \\
\hline $\begin{array}{l}\text { Lumpkin, et al. } \\
\text { (2013) }\end{array}$ & $\begin{array}{l}\text { Lograr una fuerte posición de ganancias sociales es uno de los pro- } \\
\text { pósitos del emprendimiento social, que involucra factores, procesos } \\
\text { y resultados. }\end{array}$ & - Beneficio social \\
\hline Urbal III (2013) & $\begin{array}{l}\text { Nace con la decisión de una o varias personas emprendedoras, que } \\
\text { va más allá de la búsqueda de un beneficio económico, su motiva- } \\
\text { ción se encuentra en crear oportunidades de empleo para terceros. } \\
\text { Es decir, persigue objetivos sociales para la mejora de la calidad de } \\
\text { vida personal y social. }\end{array}$ & $\begin{array}{l}\text { - } \quad \text { Beneficio social } \\
\text { - Objetivos sociales }\end{array}$ \\
\hline Fischel (2013) & $\begin{array}{l}\text { Trata de innovaciones que tienen impacto significativo en los ámbitos } \\
\text { social y ambiental, diseñadas a partir de métodos empresariales. Es } \\
\text { decir, se apoya en la disciplina empresarial con el fin de alcanzar una } \\
\text { misión social. }\end{array}$ & $\begin{array}{l}\text { - Innovación } \\
\text { - Misión social }\end{array}$ \\
\hline $\begin{array}{l}\text { Said Business } \\
\text { School (2014) }\end{array}$ & $\begin{array}{l}\text { Se refiere a la práctica de combinar la innovación, el ingenio y la } \\
\text { oportunidad de abordar los desafíos sociales y ambientales críticos. } \\
\text { Los emprendedores sociales se centran en transformar los sistemas } \\
\text { y las prácticas que son las causas fundamentales de la pobreza, la } \\
\text { marginación, el deterioro ambiental y la consiguiente pérdida de } \\
\text { la dignidad humana. Al hacerlo, pueden configurarse con fines de } \\
\text { lucro o sin fines de lucro, y en ambos casos, su objetivo principal es la } \\
\text { creación de cambios sostenibles de los sistemas. }\end{array}$ & $\begin{array}{l}\text { - Innovación } \\
\text { - Oportunidad } \\
\text { - Desafío social } \\
\text { - Cambios sosteni- } \\
\text { bles }\end{array}$ \\
\hline
\end{tabular}


Todos estos conceptos, permiten identificar los aspectos más importantes del emprendimiento social:

1. La misión social que pone énfasis en desarrollar bienes y servicios que cubran directamente las necesidades más apremiantes de la sociedad, creando de este modo valor social.

2. La innovación que busca la forma de cubrir las necesidades con proyectos innovadores o soluciones nuevas que no hayan sido aplicadas anteriormente.

3. Los beneficios, es decir, conseguir la sostenibilidad financiera de los proyectos que emprenden.

4. La búsqueda y aprovechamiento de oportunidades para resolver necesidades sociales de los grupos menos favorecidos.

5. Asumir riesgos, se proponen y desarrollan proyectos que en principio no van a proporcionar resultados positivos, pero que a largo plazo llegan a ser autosostenibles.

6. Buscan un cambio social a través del establecimiento de un equilibrio en el uso de los recursos de la sociedad.

Las variables contenidas en el concepto de emprendimiento social son la misión social, la innovación, la sostenibilidad financiera, la solución de necesidades sociales, la aceptación de riesgos y el cambio social.

\section{Emprendedor social}

El emprendedor social ofrece un cambio innovador en los negocios con inteligencia y alcanza objetivos con una valiosa contribución al desarrollo social. Baber, Jain y Baber (2012) apuntan a que para el desarrollo de nuestra sociedad surge la necesidad de que este empresario asuma riesgos calculados y posibilite la creación de una sociedad autosuficiente con pasión, innovación, creatividad, impulso y cambio del mundo, constituyéndose así en la parte más activa del proceso de cambio social. Se caracteriza por ser un empresario educado, que comprende fácilmente los problemas y también es consciente 
de cómo erradicarlos, y posee habilidades e inteligencia de todas las técnicas modernas para convertir todas las cosas improductivas en productivas.

Uno de los emprendedores mundialmente conocido es Muhammad Yunus, Premio Nobel de Paz 2006, un economista excepcional y empresario que reconoció la difícil situación de sus compatriotas en Bangladesh: a pesar de su trabajo duro, no ganaban salarios suficientes y seguían viviendo en la pobreza. Yunus creía que esa pobreza era consecuencia de la falta de acceso a los recursos necesarios para identificar y explotar oportunidades, particularmente recursos financieros. Para corregir el desequilibrio de la distribución de la riqueza, Yunus proporcionó pequeñas sumas de crédito - microfinanzas - como un mecanismo para ayudar a aprovechar las oportunidades; creía que si se utilizaban las microfinanzas para apoyar el inicio de la sostenibilidad social de empresas que abordaran los problemas sociales, ambientales y de otro tipo creados por causa de la pobreza y la falta de acceso a las oportunidades, la estructura del mercado capitalista podría ser ensanchada; esto a su vez crearía oportunidades para la explotación del mercado que estaría disponible para una gama más diversa de empresarios, incluidos los emprendedores sociales. Convencido por este argumento, Yunus estableció el Grameen Bank en 1983 y se convirtió en un emprendedor social. Su banco, que proporciona microcréditos a personas socialmente desfavorecidas que carecen de garantías, ha revolucionado la industria del crédito e iniciado el crecimiento de la inversión internacional en el sector del microcrédito, fomentando el fenómeno de la empresa social a escala mundial (Danko, Brunner \& Kraus, 2011).

A continuación, se presentan algunos puntos que revelan claramente la importancia de un emprendedor en el desarrollo social (Baber, Jain \& Baber, 2012):

- Oportunidad de empleo

- Desarrollo de sectores atrasados

- Distribución equitativa de la riqueza

- Reducción de la brecha entre áreas rurales y urbanas

- Mejora de la calidad de vida

- Utilización óptima de recursos

- Conservación del patrimonio

- Contribución al crecimiento económico de las naciones

- Satisfacción social 


\section{De estos puntos se puede inferir que los emprendedores sociales son importantes para}

\section{el desarrollo económico y social de los países.}

\section{La tabla 2 muestra la revisión de los conceptos de emprendedor social que han surgido de la revisión de la literatura.}

Tabla 2. Conceptos de emprendedor social

\begin{tabular}{|c|c|c|}
\hline Autor/año & Concepto & Variables \\
\hline $\begin{array}{l}\text { Waddock y Post } \\
\text { (1991) }\end{array}$ & $\begin{array}{l}\text { Son ciudadanos particulares que juegan un rol crítico en el } \\
\text { logro de cambios catalíticos en la agenda del sector público } \\
\text { y la percepción de los problemas sociales. }\end{array}$ & $\begin{array}{l}\text { - Percepción de problemas } \\
\text { sociales }\end{array}$ \\
\hline Thake y Zadek (1997) & $\begin{array}{l}\text { Están impulsados por un deseo de justicia social. Buscan una } \\
\text { relación directa entre sus acciones y una mejora en la calidad } \\
\text { de vida de las personas con las que trabajan y los que tratan de } \\
\text { servir. Su objetivo es producir soluciones que sean sostenibles } \\
\text { financiera, organizativa, social y ambientalmente. }\end{array}$ & $\begin{array}{l}\text { - Deseo de justicia social } \\
\text { - Soluciones sostenibles }\end{array}$ \\
\hline Boschee (1998) & $\begin{array}{l}\text { Son ejecutivos sin ánimo de lucro que prestan cada vez más } \\
\text { atención a las fuerzas del mercado sin perder de vista sus } \\
\text { misiones fundamentales, balanceando imperativos morales } \\
\text { y la obtención de beneficios y que ese equilibrio sea el cora- } \\
\text { zón y el alma del movimiento. }\end{array}$ & $\begin{array}{l}\text { Equilibrio entre moral y } \\
\text { beneficios }\end{array}$ \\
\hline Dees (1998) & $\begin{array}{l}\text { Desempeñan el papel de agentes de cambio en el sector } \\
\text { social a través de (1) la adopción de una misión para crear y } \\
\text { mantener valor social; (2) el reconocimiento y la implacable } \\
\text { búsqueda de nuevas oportunidades para servir a esa misión; } \\
\text { (3) la realización de un proceso de continua innovación, } \\
\text { adaptación y aprendizaje; (4) la actuación con valentía y sin } \\
\text { estar limitado por los recursos actualmente disponibles, y (5) } \\
\text { la responsabilidad sobre los beneficiarios atendidos y por } \\
\text { los resultados creados. }\end{array}$ & 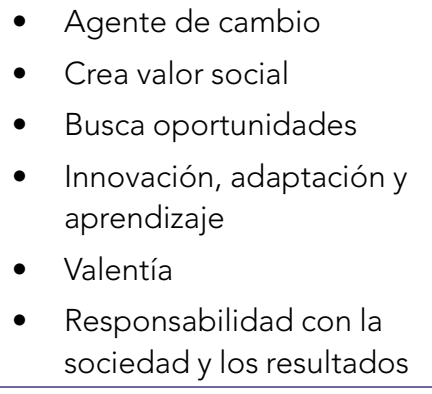 \\
\hline Reis (1999) & $\begin{array}{l}\text { Crean valor social a través de la innovación y el aprovecha- } \\
\text { miento de los recursos financieros para el desarrollo social, } \\
\text { económico y comunitario. }\end{array}$ & $\begin{array}{l}\text { - } \quad \text { Crea valor social } \\
\text { - Innovación } \\
\text { - Aprovecha recursos }\end{array}$ \\
\hline Prabhu (1999) & $\begin{array}{l}\text { Son personas que crean y gestionan las organizaciones em- } \\
\text { presariales o empresas innovadoras, cuya misión principal es } \\
\text { el cambio social y el desarrollo de su grupo de clientes. }\end{array}$ & $\begin{array}{l}\text { - Innovación } \\
\text { - Cambio y desarrollo social }\end{array}$ \\
\hline De Leeuw (1999) & $\begin{array}{l}\text { Considera que los emprendedores sociales son mediadores } \\
\text { excepcionales capaces de liderar a una amplia gama de } \\
\text { individuos diferentes, motivándolos a realizar actividades } \\
\text { colectivas que deriven en resultados sociales positivos. }\end{array}$ & $\begin{array}{l}\text { - } \text { Mediador } \\
\text { - } \quad \text { Líder } \\
\text { - Resultado social }\end{array}$ \\
\hline Brinckerhoff (2000) & $\begin{array}{l}\text { Son personas que asumen riesgos en nombre de las perso- } \\
\text { nas a las que sirve su organización. }\end{array}$ & - Asumen riesgos \\
\hline
\end{tabular}




\begin{tabular}{|c|c|c|}
\hline Autor/año & Concepto & Variables \\
\hline $\begin{array}{l}\text { Thompson, et al. } \\
\text { (2000) }\end{array}$ & $\begin{array}{l}\text { Son las personas que se dan cuenta de que hay una oportu- } \\
\text { nidad para satisfacer alguna necesidad no cubierta puesto } \\
\text { que el sistema de asistencia social del Estado no quiere o } \\
\text { no puede cumplir, y que reúnen los recursos necesarios y los } \\
\text { utilizan para cubrir esa necesidad. }\end{array}$ & $\begin{array}{l}\text { - } \quad \text { Detecta oportunidad } \\
\text { - Cubren necesidades }\end{array}$ \\
\hline Brinkerhoff (2001) & $\begin{array}{l}\text { Son personas que constantemente están en busca de nue- } \\
\text { vas formas de servir a sus beneficiarios y añadir valor a los } \\
\text { servicios existentes. }\end{array}$ & $\begin{array}{l}\text { - Innovador social } \\
\text { - Añade valor }\end{array}$ \\
\hline Labarre et al. (2001) & $\begin{array}{l}\text { Son innovadores dedicados, decididos a abordar algunos } \\
\text { de los desafíos más profundos de la sociedad mediante la } \\
\text { adopción de nuevas ideas de negocio. }\end{array}$ & $\begin{array}{l}\text { - Innovador } \\
\text { - Asume riesgos }\end{array}$ \\
\hline $\begin{array}{l}\text { Guclu, Dee y } \\
\text { Anderson (2002) }\end{array}$ & $\begin{array}{l}\text { Comienzan con la visión de una oportunidad atractiva con } \\
\text { suficiente potencial de tener un impacto social positivo para } \\
\text { justificar la inversión de tiempo, energía y dinero necesarios } \\
\text { para perseguirla. }\end{array}$ & $\begin{array}{l}\text { - Visionario } \\
\text { - } \text { Detecta oportunidad } \\
\text { - } \text { Busca impacto social }\end{array}$ \\
\hline $\begin{array}{l}\text { Morse y Dudley } \\
\text { (2002) }\end{array}$ & $\begin{array}{l}\text { Son aquellos que combinan el espíritu de empresa y de la } \\
\text { comunidad para construir capital social en el proceso de } \\
\text { mejoramiento de la comunidad. }\end{array}$ & - Construyen capital social \\
\hline Drayton (2002) & $\begin{array}{l}\text { Son agentes de cambio importantes, cuyo núcleo de valores } \\
\text { se centra en identificar, abordar y resolver problemas sociales. }\end{array}$ & $\begin{array}{l}\text { - Agentes de cambio } \\
\text { - Resuelve problemas } \\
\text { sociales }\end{array}$ \\
\hline Thompson (2002) & $\begin{array}{l}\text { Personas con cualidades y comportamientos asociados con } \\
\text { el empresario de negocios que operan en la comunidad y } \\
\text { están más preocupados por cuidar y ayudar que por hacer } \\
\text { dinero. }\end{array}$ & $\begin{array}{l}\text { - Empresario comunitario } \\
\text { - Bienestar social }\end{array}$ \\
\hline $\begin{array}{l}\text { Sullivan, Weerawa y } \\
\text { Carnegie (2003) }\end{array}$ & $\begin{array}{l}\text { Están impulsados por la misión social de creación de un } \\
\text { mejor valor social que sus competidores lo que se traduce } \\
\text { en que exhiben un comportamiento virtuoso empresarial- } \\
\text { mente. En segundo lugar, exhiben un juicio equilibrado, } \\
\text { una unidad coherente de propósitos y de acción frente a la } \\
\text { complejidad. En tercer lugar, exploran y reconocen las opor- } \\
\text { tunidades para crear un mejor valor social para sus clientes. } \\
\text { Por último, los empresarios sociales muestran la capacidad } \\
\text { de innovación, proactividad y asunción de riesgos en la } \\
\text { toma de decisiones clave. }\end{array}$ & $\begin{array}{l}\text { - } \text { Crean valor social } \\
\text { - Juicio equilibrado } \\
\text { - Reconoce oportunidades } \\
\text { - Capacidad de innovación } \\
\text { - Proactividad } \\
\text { - Asume riesgos }\end{array}$ \\
\hline $\begin{array}{l}\text { Alford, Brown y Letts } \\
\text { (2004) }\end{array}$ & $\begin{array}{l}\text { Crea soluciones innovadoras a los problemas sociales inme- } \\
\text { diatos y moviliza las ideas, las capacidades, los recursos y los } \\
\text { acuerdos sociales necesarios para llevar a cabo transforma- } \\
\text { ciones sociales. }\end{array}$ & $\begin{array}{l}\text { - Innovación social } \\
\text { - Transforma la sociedad }\end{array}$ \\
\hline Bornstein (2004) & $\begin{array}{l}\text { Es un innovador con una nueva idea poderosa, que combina } \\
\text { lo visionario con la creatividad en la resolución de proble- } \\
\text { mas reales, que tiene una fibra ética fuerte y está totalmente } \\
\text { poseído por su visión para el cambio. }\end{array}$ & $\begin{array}{l}\text { - Innovador } \\
\text { - } \text { Creativo } \\
\text { - Ético } \\
\text { - Visión para el cambio }\end{array}$ \\
\hline Harding (2004) & $\begin{array}{l}\text { Empresarios motivados por objetivos sociales para instigar } \\
\text { algún tipo de nueva actividad o emprendimiento. }\end{array}$ & - Objetivos sociales \\
\hline
\end{tabular}




\begin{tabular}{|c|c|c|}
\hline Autor/año & Concepto & Variables \\
\hline $\begin{array}{l}\text { PBS The New Heroes } \\
\text { (2005) }\end{array}$ & $\begin{array}{l}\text { Identifica y resuelve los problemas sociales a gran escala. } \\
\text { Del mismo modo que los emprendedores de negocios } \\
\text { crean y transforman industrias enteras, los emprendedores } \\
\text { sociales actúan como agentes de cambio para la sociedad, } \\
\text { aprovechan las oportunidades que otros pasan por alto con } \\
\text { el fin de mejorar los sistemas; inventan y difunden nuevos } \\
\text { enfoques y promueven soluciones sostenibles que generan } \\
\text { valor social. }\end{array}$ & $\begin{array}{l}\text { - } \text { Identifica necesidades } \\
\text { - } \text { - Ageciales } \\
\text { - Innovación } \\
\text { - Valor social }\end{array}$ \\
\hline Light (2006) & $\begin{array}{l}\text { Individuo, grupo, red, organización o alianza de organizacio- } \\
\text { nes que busca un cambio sostenible a gran escala a través } \\
\text { de las ideas innovadoras para hacer frente a los problemas } \\
\text { sociales significativos ya sea realizado por gobiernos, orga- } \\
\text { nizaciones no lucrativas o empresas. }\end{array}$ & $\begin{array}{l}\text { - Cambio sostenible } \\
\text { - Innovación social }\end{array}$ \\
\hline Zahra, et al. (2009) & $\begin{array}{l}\text { Son agentes que, debido a su motivación, crean valor sin } \\
\text { preocuparse por las ganancias. }\end{array}$ & - Creación de valor social \\
\hline $\begin{array}{l}\text { Melián y Campos } \\
\text { (2010) }\end{array}$ & $\begin{array}{l}\text { Forman empresas cuyos fines no son el lucro o al menos no } \\
\text { lo son de forma prioritaria y maximizadora. Surge del auto- } \\
\text { empleo colectivo y no del individual, favorece la integración } \\
\text { de lo social y lo económico en su empresa y se dota de } \\
\text { mecanismos de control y coordinación basados en la partici- } \\
\text { pación democrática. }\end{array}$ & $\begin{array}{l}\text { - Autoempleo colectivo } \\
\text { - Integración social } \\
\text { - Participación democrática }\end{array}$ \\
\hline Curto (2012) & $\begin{array}{l}\text { Presenta cinco características fundamentales: tiene como } \\
\text { objetivo crear valor social, es capaz de captar necesidades } \\
\text { sociales, contraataca con propuestas innovadoras, su } \\
\text { aversión al riesgo está por debajo de la media y dispone de } \\
\text { escasos recursos para llevar a cabo su labor. }\end{array}$ & $\begin{array}{l}\text { - Crea valor social } \\
\text { - Capta necesidades } \\
\text { sociales } \\
\text { - Innovación } \\
\text { - Pocos recursos }\end{array}$ \\
\hline $\begin{array}{l}\text { Salinas y Osorio } \\
(2012)\end{array}$ & $\begin{array}{l}\text { Tiene una iniciativa personal con proyección comunitaria } \\
\text { que desarrolla en contextos sociales y económicos generan- } \\
\text { do sinergias cooperativas entre los diferentes actores que } \\
\text { participan en toda acción emprendedora. }\end{array}$ & $\begin{array}{l}\text { - } \quad \text { Proyección comunitaria } \\
\text { - } \quad \text { Sinergias cooperativas }\end{array}$ \\
\hline Fischel (2013) & $\begin{array}{l}\text { Persona con alta vocación social, con inquietudes intelectua- } \\
\text { les y espíritu innovador, que sale de los marcos establecidos } \\
\text { y asume riesgos, con el fin de alcanzar metas en lo social, } \\
\text { económico y ambiental. Aprovechan óptimamente los } \\
\text { recursos humanos y materiales que ofrece el ámbito local, a } \\
\text { través de la identificación de sinergias, alianzas estratégicas y } \\
\text { encadenamientos. }\end{array}$ & $\begin{array}{l}\text { - } \text { Vocación social } \\
\text { - } \\
\text { - } \text { Aspíritu innovador } \\
\text { - Identifica sinergias }\end{array}$ \\
\hline Ashoka (2014) & $\begin{array}{l}\text { Son personas con soluciones innovadoras a los problemas } \\
\text { sociales más acuciantes de la sociedad. Son ambiciosos y } \\
\text { persistentes, al abordar los principales problemas sociales y } \\
\text { ofrecer nuevas ideas para el cambio a gran escala. }\end{array}$ & $\begin{array}{l}\text { - Innovadores } \\
\text { - Ambiciosos } \\
\text { - Persistentes } \\
\text { - Aborda problemas so- } \\
\text { ciales }\end{array}$ \\
\hline NYU Stern (2014) & $\begin{array}{l}\text { Están transformando el panorama empresarial y social } \\
\text { mediante la aplicación de la disciplina y los principios de ne- } \\
\text { gocios para abordar algunos de los problemas más difíciles } \\
\text { del mundo. }\end{array}$ & $\begin{array}{l}\text { - Transformadores } \\
\text { - Disciplinados } \\
\text { - Enfoque social }\end{array}$ \\
\hline
\end{tabular}




\begin{tabular}{|c|c|c|}
\hline Autor/año & Concepto & Variables \\
\hline $\begin{array}{l}\text { Schwab Foundation } \\
\text { (2014) }\end{array}$ & $\begin{array}{l}\text { Es un visionario pragmático que alcanza un cambio social a } \\
\text { gran escala, sistémico y sostenible a través de un nuevo in- } \\
\text { vento, un enfoque diferente, una aplicación más rigurosa de } \\
\text { las tecnologías o estrategias conocidas, o una combinación } \\
\text { de estos. Se centra ante todo en la creación de valor social y } \\
\text { trata de optimizar la creación de valor económico. }\end{array}$ & $\begin{array}{l}\text { - Cambio social } \\
\text { - Visionario } \\
\text { - Pragmático } \\
\text { - Innovador social } \\
\text { - Valor social }\end{array}$ \\
\hline $\begin{array}{l}\text { Skoll Foundation } \\
\text { (2014) }\end{array}$ & $\begin{array}{l}\text { Son agentes de cambio de la sociedad: creadores de inno- } \\
\text { vaciones que alteran el status quo y cambian nuestro mundo } \\
\text { para mejor. Crean modelos innovadores que pueden desen- } \\
\text { cadenar un cambio a gran escala de los problemas sociales } \\
\text { aparentemente insolubles. }\end{array}$ & $\begin{array}{l}\text { - } \\
\text { - } \quad \text { Innonte de cambio } \\
\text { - }\end{array}$ \\
\hline
\end{tabular}

Con base en la revisión anterior se puede señalar que los emprendedores sociales ayudan en la creación de valores sociales y económicos. Ellos desempeñan un papel importante en la generación de empleo, el desarrollo económico, la innovación y la formación de capital social y financiero. Los emprendedores sociales son necesarios ya que buscan oportunidades para resolver los problemas que prevalecen en la sociedad y, al descubrir las causas, a través de sus ideas y creaciones innovadoras, eliminan esos problemas y, sobre todo, obtienen ganancias. Las principales variables contenidas en el concepto de emprendedor social son fomentar valores sociales y económicos, resolver problemas sociales, creativos, innovadores, persistentes, éticos y agentes de cambio.

\subsection{Las motivaciones del emprendedor social}

Roberts y Woods (2005) identifican como factores diferenciadores entre empresarios comerciales y sociales la motivación (tabla 3); la de los emprendedores sociales no está orientada a los beneficios, su motivación está impulsada por la necesidad de abordar las cuestiones sociales, sociopolíticas y las primarias fundamentales de aquellos que sufren carencias. Un aspecto importante del perfil del emprendedor social es la razón que lo impulsa a realizar un emprendimiento para cubrir una necesidad social (Seham et al. 2017). 
Tabla 3. Motivaciones del emprendedor social

\begin{tabular}{|c|c|}
\hline Tomar la iniciativa para iniciar & Tener la persistencia para continuar \\
\hline Los problemas y retos sociales actuales & Redes sociales \\
\hline El deseo de resolver las necesidades sociales insatisfechas. & - Las redes sociales proporcionan refuerzos para \\
\hline El deseo de cambiar la sociedad, lo que lleva a identificar & guiar el comportamiento humano. \\
\hline oportunidades y encontrar soluciones innovadoras. & - Los emprendedores sociales reciben estímulo, \\
\hline Inspiración & soporte y fuerza de manejo. \\
\hline $\begin{array}{l}\text { La exposición a diferentes situaciones y experiencias que } \\
\text { inspiran nuevas ideas y visiones. }\end{array}$ & $\begin{array}{l}\text { - Las redes sociales proporcionan apoyo emocional, } \\
\text { racional y físico a los emprendedores sociales. }\end{array}$ \\
\hline $\begin{array}{l}\text { Las experiencias en el extranjero o heredadas de creencias } \\
\text { religiosas pueden ser una fuente de inspiraciones }\end{array}$ & $\begin{array}{l}\text { - Las redes sociales ayudan a los empresarios a obte- } \\
\text { ner acceso a las finanzas. }\end{array}$ \\
\hline Experiencia personal previa & \multirow{3}{*}{$\begin{array}{l}\text { - Las redes sociales brindan habilidades y conoci- } \\
\text { mientos para que las organizaciones prosperen. }\end{array}$} \\
\hline $\begin{array}{l}\text { Las experiencias personales de los empresarios sociales } \\
\text { pueden conducirlos a iniciar proyectos sociales. }\end{array}$ & \\
\hline $\begin{array}{l}\text { Diferentes experiencias incitan a los empresarios hacia la } \\
\text { búsqueda del bienestar y la felicidad de otros. }\end{array}$ & \\
\hline
\end{tabular}

Fuente: elaborado a partir de Seham et al. (2017).

Como se puede ver en la tabla 3, la motivación emana de diversas fuentes que tienen como denominador común la identificación de necesidades sociales ya sea por experiencia propia o de terceros y el deseo de resolverlas.

\subsection{Características de los emprendedores sociales}

El emprendimiento social implica no solo la implementación de ideas innovadoras, también incluye un estilo de liderazgo extraordinario por parte de los empresarios (Danko, Brunner \& Kraus, 2011). 
Tabla 4. Características de los emprendedores sociales

\begin{tabular}{ll}
\hline Tomar la iniciativa para empezar & Tener la persistencia para continuar \\
\hline Mentalidad empresarial & Perseverancia \\
Experiencia de trabajo previa & La capacidad para superar la adversidad co- \\
Conocimientos, habilidades y know-how (saber cómo) & la burocracia, la corrupción y la ausencia \\
Innovación & de apoyo gubernamental. \\
Crear nuevos productos/servicios & Superar la ausencia de estructura legal, \\
Entrega & apoyo financiero, la cultura, la innovación y \\
Procesos y modelos de negocio desafios externos y larreras. \\
Aspectos compasivo y humanitario & \\
Impulsado por la compasión y la responsabilidad social & \\
Empatía y comprensión de las recompensas de ganancia de beneficiar & \\
a los demás & \\
La aceptación de riesgos & \\
Desafíos incrustados en las normas culturales & \\
Enfrentar incertidumbres y riesgos adicionales & \\
Enfrentar la inestabilidad política y económica & \\
\hline
\end{tabular}

Fuente: elaborada a partir de Seham et al. (2017).

\section{Conclusiones}

Los temas de emprendedor y emprendimiento social han sido poco estudiados en el contexto Latinoamericano; la revisión de la literatura que se ha realizado en este trabajo pretende servir de marco de referencia para impulsar la realización de estudios en este tópico.

El emprendimiento social persigue la misión social de desarrollar bienes y servicios para cubrir las necesidades de los sectores menos favorecidos de la sociedad (creación de valor social); busca soluciones innovadoras que no han sido aplicadas y genera proyectos para llevarlos a cabo y en muchos casos no tienen un costos (innovación social); administra de modo eficiente los recursos financieros, humanos y materiales con el fin de generar beneficio financiero (sostenibilidad financiera); busca y aprovecha las oportunidades para resolver necesidades sociales con el fin de establecer un equilibrio social (visión social), y asume riesgos desarrollando proyectos que en un inicio no serán rentables (rentabilidad social). 
El emprendedor social es impulsado por un deseo de justicia social (vocación social), tiene la habilidad de percibir los problemas sociales (visión social); busca soluciones sostenibles, procurando el equilibrio moral y la obtención de beneficios (ética social); incursiona como un agente de cambio que asume riesgos y crea valor social (cambio social). Además, aprovecha las oportunidades y los recursos para afrontar las necesidades a través de la innovación social (riesgo social); tiene la capacidad de gestionar, aplicando un liderazgo democrático y construyendo un capital social (emprendimiento sostenible).

Es necesario impulsar los estudios en estos temas para conocer de qué manera el emprendimiento social se está desarrollando en el contexto latinoamericano y así determinar cómo, desde el ámbito académico, se pueden apoyar estos procesos a través de la vinculación empresa-universidad-sociedad.

\section{Referencias}

Aguilera, R. V., Rupp, D. E., Williams, C. A., \& Ganapathi, J. (2007). Putting the S back in corporate social responsibility: A multilevel theory of social change in organizations. Academy of Management Review, 32(3), 836-863. https://doi.org/10.2307/20159338

Alford, S. H., Brown, L. D., \& Letts, C. W. (2004). Social entrepreneurship: Leadership that facilitates societal transformation. Working. Cambridge, MA: Center for Public Leadership, John F. Kennedy School of Government.

Ashoka (2014). Todo el mundo puede ser impulsor de cambios. Recuperado de http://spain. ashoka.org/sites/spainsix/files/One\%20Pager\%20Ashoka\%20\%28Espa\%C3\%B1ol\%29.pdf

Austin, J. E., Stevenson, H., \& Wei-Skillern, J. (2006). Social and commercial entrepreneurship: The same, different or both? Entrepreneurship Theory and Practice, 30(1), 1-22. Recuperado de https://doi.org/10.1111/j.1540-6520.2006.00107.x

Baber, R, Jain, S, \& Baber, P. (2012). Social entrepreneurs: Changing the face of society. Recuperado de https://www.researchgate.net/publication/236003969_Social_ Entrepreneurs_Changing_the_face_of_Society

Bornstein, D. (2004). How to change the world: Social entrepreneurship and the power of ideas. Oxford University Press.

Boschee, J. (1998). Merging mission and money: A board member's guide to social entrepreneurship. Washington: National Center for Nonprofit Boards. 
Brinckerhoff, P. C. (2000). Social entrepreneurship: The art of mission-based venture development. New York: John Wiley \& Sons.

Brinkerhoff, P. (2001). Why you need to be more entrepreneurial - A how to get started. Nonprofit World, 19(6), 12-15.

Bronn, P. S., \& Vidaver-Cohen, D. (2009). Corporate motives for social initiative: Legitimacy, sustainability, or the bottom line? Journal of Business Etbics, 87, 91-109. https://doi. org/10.1007/s10551-008-9795-z

Canadian Centre for Social Entrepreneurship. (2001). Social entrepreneurship discussion paper No. 1. Recuperado de http://citeseerx.ist.psu.edu/viewdoc/download;jsessionid= 3ED60368F90C56C04EF0435EC4E7C015?doi=10.1.1.19 4.4683\&rep=rep1\&type=pdf

Chell, E. (2007). Social enterprise and entrepreneurship: towards a convergent theory of the entrepreneurial process. International Small Business Journal, 25(1), 5-26. https://doi. org/10.1177/0266242607071779

Conway, M., \& Kalakay, J. (2016). The winding road of social entrepreneurship definitions: a systematic literature review. Social Enterprise Journal, 12(2),131-160. https://doi. org/10.1108/SEJ-06-2015-0016

Cook, B., Dodds, C., \& Mitchell, W. (2003). Social entrepreneurship: false premises and dangerous forebodings. Australian Journal of Social Issues, 38(1), 57-72. https://doi. org/10.1002/j.1839-4655.2003.tb01135.x

Curto, G. (2012). Los emprendedores sociales: innovación al servicio del cambio social. Cuadernos de la Cátedra "la Caixa" de Responsabilidad Social de la Empresa y Gobierno Corporativo, (13).

Dacin, M. T., Dacin, P. A., \& Tracey, P. (2011). Social entrepreneurship: A critique and future directions. Organization Science, 22(5), 1203-1213.

Danko, A., Brunner, C. \& Kraus, S. (2011). Social entrepreneurship - An overview of the current state of research. European Journal of Management, 11(1), 82-91.

De Leeuw, E. (1999). Healthy cities: Urban social entrepreneurship for health. Health Promotion International, 14(3), 261-269. https://doi.org/10.1093/heapro/14.3.261

Dees, J. G. (1998). The meaning of social entrepreneurship. Comments and suggestions contributed from the Social. Recuperado de https://centers.fuqua.duke.edu/case/wp-content/ uploads/sites/7/2015/03/Article_Dees_MeaningofSocialEntrepreneurship_2001.pdf

Dees, J. G. (2012). A tale of two cultures: Charity, problem solving and the future of social entrepreneurship. Journal of Business Ethics, 111(3), 321-334. 
De Pablo López, I. (ocubre, 2005). El emprendizaje social: motor de desarrollo y cohesión social. V Seminario sobre creación de empresas y entorno, Trujillo, Universidad Autónoma de Madrid.

Drayton, W. (2002). The citizen sector: Becoming as entrepreneurial and competitive as business. California Management Review, 44(3), 120-132.

Felix, M., Husted, B., \& Aigner, D. (2017). Opportunity discovery and creation in social entrepreneurship: An exploratory study in Mexico. Journal of Business Research, 81, 212-220.

Fischel, A. (2013). ¿Cómo educar en emprendedurismo social y ética? Congreso Internacional de Emprendedurismo Social, Universidad de Costa Rica, 6 y 7 de junio.

Fuqua School (2005). What is Social Entrepreneurship? Recuperado de http://www.caseatduke.org/ about/whatissocialentrepreneurship/

Fowler, A. (2000). NGDOs as a moment in history: beyond aid to social entrepreneurship or civic innovation? Third World Quarterly, 21(4), 637-656.

Gartner, W. (1985). A conceptual framework for describing the phenomenon of new venture creation. The Academy of Management Review, 10(4), 696-706. Recuperado de http:// www.jstor.org/stable/258039

Gartner, W. (1989). Who is an entrepreneur? Is the wrong question. American. https://doi. org/10.1177/104225878801200401.

Guclu, A., Dees, J. G., \& Anderson, B. B. (2002). The process of social entrepreneurship: Creating opportunities worthy of serious pursuit. Durham: Duke University.

Guzmán, A., \& Trujillo, M. (2008). Emprendimiento social - Revisión de literatura. Estudios Gerenciales, 24(109), 105-125.

Harding, R. (2004). Social enterprise: the new economic engine? Business Strategy Review, 15(4), 39-43.

Haugh, H. (2005). A research agenda for social entrepreneurship. Social Enterprise Journal, 1(1), 1-12.

Hibbert, S. A., Hogg, G., \& Quinn, T. (2002). Consumer response to social entrepreneurship: The case of the Big Issue in Scotland. International Journal of Non-profit and Voluntary Sector Marketing, 7(3), 288-301.

Johnson, S. (2000). Literature review on social entrepreneurship. Canadian Center for Social Entrepreneurship.

Korosec, R. L., \& Berman, E. M. (2006). Municipal support for social entrepreneurship. Public Administration Review, 66(3), 448-462. 
Kostetska, I., \& Berezyak, I. (2014). Social entrepreneurship as an innovative solution mechanism of social problems of society. Management Theory and Studies for Rural Business and Infrastructure Development. 36(3), 569-577.

Kuratko, D. (2005). The emergence of entrepreneurship education: Development, trends, and challenges. Entrepreneurship Theory and Practice, 29(5), 577-598.

Labarre, P., Fishman, C., Hammons, K.H., \& Warner, F. (2001). Who's fast leaders 2002. Fast Company, 52, 83-128.

Leadbeater, C. (1997). The rise of the social entrepreneur. Londres: Demos.

Lepoutre, J., Justo, R., Terjesen, S., \& Bosma, N. (2011). Designing a global standardized methodology for measuring social entrepreneurship activity: The global entrepreneurship monitor social entrepreneurship study. Small Business Economic 40, 693-714. https://doi. org/10.1007/s11187-011-9398-4

Light, P. C. (2006). Reshaping social entrepreneurship. Stanford Social Innovation Review, $4(3), 47-51$.

Lumpkin G. T., Moss T. W., Gras D. M., Kato S., \& Amezcua, A. (2013). Entrepreneurial processes in social contexts: how are they different, if at all? Small Business Economics, 40(3), 761-783.

MacMillan, I. A. (2003). Social entrepreneurs: playing the role of change agents in society. Recuperado de http://knowledge.wharton.upenn.edu/article/socialentrepreneurs-playing-the-role-of-change-agents-in-society/

Mair, J., \& Marti, I. (2006). Social entrepreneurship research: A source of explanation, prediction and delight. Journal of World Business, 41, 36-44.

Mair, J., \& Noboa, E. (2006). Social entrepreneurship: how intentions to create a social enterprise get formed. En J. Mair, J. Robinson, \& K. Hockerts (Eds.), Social entrepreneurship, pp.89-94. London: Palgrave Macmillan.

Mair, J., \& Schoen, O. (2007). Successful social entrepreneurship business models in the context of developing economies. International Journal of Emerging Markets, 2(1),54-68.

Martin, R. L., \& Osberg, S. (2007). Social entrepreneurship: The case for definition. Stanford Social Innovation Review, 5(2), 28-39.

Melián, A. \& Campos, V. (2010). Emprendedurismo y economía social como mecanismos de inserción sociolaboral en tiempos de crisis. Revesco, 100, 43-67.

Morse, R., \& Dudley, L. (2002). Civic entrepreneurs and collaborative leadership. PA Times, 25(8), 2. 
Nicholls, A. (2006). Playing the field: A new approach to the meaning of social entrepreneurship. Social Enterprise Journal, 2(1), 1-5.

NYU Stern (2014). Creating social change \& impact through business innovation. Recuperado de http://www.stern.nyu.edu/experience-stern/about/departmentscenters-initiatives/centers-of-research/berkley-center/programs/social-entrepreneurship.

Pache, A. C., \& Santos, F. (2013). Inside the hybrid organization: Selective coupling as a response to competing institutional logics. Academy of Management Journal, 56, 972-1001.

PBS The New Heroes (2005). What is social entrepreneurship? Recuperado de http://www. pbs.org/opb/thenewheroes /whatis/

Peredo, A. M., \& McLean, M. (2006). Social entrepreneurship: a critical review of the concept. Journal of World Business, 41(1), 56-65.

Prabhu, G. N. (1999). Social entrepreneurship leadership. Career Development International, $4(3), 140-145$.

Reis, T. (1999). Unleashing the new resources and the entrepreneurship for the common good: A scan synthesis and scenario for action. W. K. Kellogg foundation Battle Creek.

Rey, A., Ribeiro, D., \& Sánchez, J. (2016). Giving back to society: Job creation through social entrepreneurship. Journal of Business Research, 69, 2067-2072.

Rodríguez L. (2007). Emprendimientos sociales: Una alternativa para la inserción social y laboral en el estado de Oaxaca. (Tesis de pregrado, México, unam).

Rodríguez, D. (2016). Emprendimiento sostenible, significado y dimensiones. Revista Katharsis, (21), 419-448. Recuperado de http://revistas.iue.edu.co/index.php/katharsis

Roberts, D., \& Woods, C. (2005). Changing the world on a shoestring: the concept of social entrepreneurship. University of Auckland Business Review, 19(1), 45-51.

Said Business School (2014). Defining social entrepreneurship. Recuperado de http://www. sbs.ox.ac.uk/ideas-impact/skoll/about-skoll-centre-social-entreprene urship/what-social-entrepreneurship

Salinas, F., \& Osorio, L. (2012). Emprendimiento y Economía social, oportunidades y efectos en una sociedad en transformación. Revista de Economía Pública, Social y Cooperativa, 75, 129-151.

Seelos, C., \& Mair, J. (2005). Social entrepreneurship: creating new business models to serve the poor. Business Horizons, 48(3), 241-246.

Seham G., Ahmed T., \& Ayman, I. (2017). What motivates social entrepreneurs to start social ventures? An exploratory study in the context of a developing economy. Social Enterprise. Journal, 13(3), 268-298. https://doi.org/10.1108/SEJ-05-2016-0014 
Sekliuckiene, J., \& Kisielius, E. (2015). Development of social entrepreneurship initiatives: A theoretical framework. Procedia - Social and Behavioral Sciences, 213, 1015-1019.

Smallbone, D., Evans, M., Ekanem, I., \& Butters, S. (2001). Researching social Enterprise: Final report to the small business service. Centre for Enterprise and Economic Development Research. Middlesex University Business School.

Schwab Foundation (2014). What is a social entrepreneur? Recuperado de http://www.schwabfound.org/content/what-social-entrepreneur.

Selamé T. (1999). Emprendimiento juvenil. Santiago de Chile: Universidad Santiago de Chile.

Silva, A., Botero, L., \& Soto, P. (2014). Sistematización de las experiencias de madrinazgo en la Ciudad de Medellín con las corporaciones para el desarrollo y la autonomía económica de las mujeres de atención a la primera infancia. (Tesis de maestría, Medellín, Universidad EAFIT).

Social Innovation, Entrepreneurship and Enterprise Alliance - SIEE-. (2014). Social enterprise: Doing business differently for a more inclusive society. Recuperado de https://employeeownership.com.au/eoa/wp-content/uploads/2015/08/Social_Manifesto_13.pdf

Skoll Foundation (2014). Approach y Skoll Awards: frequently asked questions. Recuperado de http://www.skollfoundation.org/

Sullivan M. G., Weerawardena, J., \& Carnegie, K. (2003). Social entrepreneurship: Towards conceptualization. International Journal of Nonprofit and Voluntary Sector Marketing, $8(1), 76-88$.

Talbot, C., Tregilgas, P., \& Harrison, K. (2002). Social enterprise in Australia: An introductory bandbook. Adelaide Central Mission.

Tan, W. L., Williams, J., \& Tan, T. M., (2005). Defining the 'social' in 'social entrepreneurship': Altruism and entrepreneurship. International Entrepreneurship and Management Journal, 1(3), 353-365.

Thake, S., \& Zadek, S. (1997). Practical people, noble causes. How to support community based social entrepreneurs. USA: New Economic Foundation

Thompson, J., Alvy, G., \& Lees, A. (2000). Social entrepreneurship - a new look at the people and the potential. Management Decision, 38(5), 328-338.

Thompson, J. L. (2002). The world of the social entrepreneur. The International Journal of Public Sector Management, 15(5), 412-431.

Thompson, J. L. (2008). Social enterprise and social entrepreneurship: where have we reached? A summary of issues and discussion points. Social Enterprise Journal, 4(2), 149-161. 
Tukamushaba, E., Orobia, L., \& George, B. (2011). Development of a conceptual model to understand international social entrepreneurship and its application in the Ugandan context. Journal of International Entrepreneurship, 9(4), 282-298. https://doi.org/10.1007/ s10843-011-0079-9

Unión Europea (2014). La iniciativa de emprendimiento social de la Comisión Europea. Bélgica: EU.

Urbal III (2013). Documento marco de reflexión sobre el emprendedurismo y la innovación social. L' Hospitalet, 27-28 de junio, Barcelona.

Waddock, S. A., \& Post, J. E. (1991). Social entrepreneurs and catalytic change. Public Administration Review, 51(5), 393-401.

Weerawardena, J., \& Sullivan Mort, G. (2006). Investigating social entrepreneurship: A multidimensional model. Journal of World Business, 41(1), 21-35.

Yunus, M. (2012). Empresa para todos. Bogotá: Editorial Norma.

Zahra, S. A., Gedajlovic, E., Neubaum, D. O., \& Shulman, J. M. (2009). A typology of social entrepreneurs: Motives, search processes and ethical challenges. Journal of business venturing, 24(5), 519-532. 